\title{
Level of Self-Efficacy of Prospective Mathematics Teachers on Competencies for Planning and Organizing Instruction
}

\author{
İsmail Şan \\ Department of Education, Faculty of Education, Inonu University, Malatya, Turkey
}

Copyright (C) 2014 Horizon Research Publishing All rights reserved.

\begin{abstract}
The purpose of this study was to determine the level of self-efficacy of prospective mathematics teachers (PMTs) on the competency "planning and organizing instruction" that is one of the teacher competencies identified in 2009 by Ministry of Education. The sample of this study consists of 111 total prospective teachers (PTs) study in mathematics department of Faculty of Science and Faculty of Education, in Inonu University, in Turkey. To determine the level of self-efficacy on teacher competencies of PMTs, "Scale for Self-efficacy on the Competence of Planning and Organizing Instruction" was prepared and used by researcher. "Subject-Specific Competencies" published by Ministry of Education (2009) was used to prepare the scale. In this study, the levels of self-efficacy of PMTs on planning and organizing instruction and the variables that affect the levels were tried to determine. According to data, the level of self-efficacy on teacher competencies of PMTs both is adequate and has some differences in terms of some demographical variables. In addition, a few new teacher competencies were identified by researcher using the PMTs' views.
\end{abstract}

Keywords Prospective Teachers, Self-Efficacy, Teacher Competencies, Teaching Mathematics

\section{Introduction}

In this century, the rapid alteration and development experienced at science and technology naturally seen on educational sciences too. However, the struggle for turning to student-centered education; teachers' role on education process is incontrovertible. Turkish National Ministry of Education (TNME) showed its consciousness about this importance by preparing the teacher competencies.

Due to metamorphosis of societies and the world, educational goals are changing. The missions and the responsibilities of Turkish Education System were announced as following: "raising people that adopt national and the universal values and solve problems; act in pursuance of national education and curriculums; learn how to learn" (DPT, 2000).

TNME and universities sometimes cooperated for increasing the quality of teachers. Research and Development Department of Education (RDDE) specified the "Profile for Modern Teacher (PFMT)" in 1999. According to RDDE, modern teacher must have the following features.

- Being qualified enough to prepare students for future,

- Dominating his/her subject area,

- Knowing students,

- Planning teaching process

- Using teaching methods and techniques according to subject will be represented.

- Communicating positively with student,

- Designing the teaching environment appropriate for students and the subjects

- Helping student to participate courses actively

- Knowing the rights and responsibilities of teachers.

However, modern teacher should;

- be active about social, cultural and sports activities,

- provide coordination between schools and other institutions,

- be able to detect disruptions of educational systems and suggest solutions for them,

- be careful for his/her fig,

- be able to produce new ideas,

- be unprejudiced,

- improve himself/herself always,

- like teaching and children,

- be respectful to the democratic values and human rights (İlhan, 2004).

In 2001, TNME notified The Council of Higher Education (CHE) about that competencies expected teachers to have. Teacher competencies classified and published in pursuance 
of PFMT. These competencies were pedagogy, general culture and subject-specific competencies.

In 2002, Commission for Teacher Competencies (CTC), accumulated for the necessity of preparing the details of teaching competencies, determined detailed 206 sub-competencies in 14 main-competencies. The framework of generic competencies on teaching was constituted of 6 main-competencies, 38 sub-competencies and 251 performance indicators. These were appropriated and insured by TNME. The main competencies are following.

A. Personal and professional values

B. Knowing students

C. Educational process

D. Monitoring and evaluating learning and development

E. Relations of school, family and society

F. Knowledge on curricular and content

On the other hand, it was noticed that competency areas had been general for teachers and pointed out the importance of defining "subject specific competencies" for all courses by academics. Thereon, TNME and universities turned their studies to defining subject-specific competencies for all courses (Şahin, 2004). After detailed studies, new competencies for all courses were defined. The competencies, which were specified for elementary level mathematics teacher, are following.

Subject-specific competencies for elementary mathematics teachers

A. Planning and designing educational process

B. Competencies on subject areas of mathematics courses

C. Improving Mathematics courses skills

D. Monitoring, evaluation and improving of teaching mathematics

E. Building cooperation with school, families and society.

F. Realizing professional improvement

The competencies can be thought as skills that a teacher must have. This obligation makes us to reorganize the curricular for training of primary mathematics teachers due to the competencies.

Similarly, a framework document for high school mathematics teachers was prepared. This study seems primary mathematics teachers' competencies. This framework has the following competencies.

Subject-specific competencies for high school mathematics teachers-framework study

A. Knowing mathematics

B. Knowing teaching mathematics

C. Attitude, faith and value towards Mathematics

D. Professional development and social works (MEB-ÖYEGM, 2009)

The performance indicators of the above competencies were prepared and high school mathematics teachers are expected to indicate 83 performance indicator. TNME has embodied and standardized its expectations from teachers by defining these indicators. In this context, teacher training institutes are expected to teach PTs to gain these competencies.

\section{Literature Review}

The self-efficacy level of PMTs on teacher competencies is worth to investigate. According to some researches (Gibson and Dembo 1984, Enochs and Riggs 1990, Bandura 1997, Özkan et al. 2002, Scholz et al. 2002, Tschannen-Moran and Hoy 1998, Yavuzer and Koç, 2002) behaviors are effected by believes. Fulfilling the tasks and responsibilities needs teacher to be well educated and to have self-efficacy about performing them. Self-efficacy is described as "belief towards his/her potential to organize, fulfill and success a task (Bandura, 1994). According to Bandura, self-efficacy is based on our abilities and necessary to reach, organize and realize a behavior (Schmitz and Schwarzer, 2000). People have optimistic or pessimistic opinions that affect them about getting ready for behavior due to their self-efficacy before acting. Also, self-efficiently people have stronger and more permanent struggle than inefficacy people (Bandura 1977, 1994, Scholz et al. 2002). A teacher's self-efficacy level affects students' motivation and success. High level self-efficacy helps teachers about to use learning methods, materials, feedbacks and to find the right classroom design (Tschannen- Moran and Hoy 2001, Özkan et al. 2002). If a teacher is persuaded that methods' yields he/she pays attention to all details of that method and diversifies feedback ways (Gibson and Dembo, 1984). The results of studies, which investigate the relation between teachers' self-efficacy level and students' academic success show that teachers' self-efficacy level affects students' success and attitude positively (Gibson and Dembo 1984, Tschannen-Moran et al. 1998). Bandura claimed that preparing a classroom environment to develop students' cognition needs a high level teaching competence and self-efficacy of teachers (Yavuzer and Koç, 2002).

The performance indicators, in other words a more detailed form of generic teacher competences are valuable for teacher training institutes. In pursuant of these competencies, in Turkey, teacher training services are forced to revise their goals and develop new curricular to train teachers. In companion with this situation, a lot of studied have been held by researchers. Şeker, Deniz \& Görgen (2005) investigated the prospective teachers' assessment of themselves, mentors and faculty lecturers in terms of teacher competencies. According to the study, PTs think themselves, mentors and faculty members are competent. Çakan (2004) compared elementary and secondary school teachers in terms of their in-class assessment activities and teacher perceptions toward their qualification levels related to 
measurement and evaluation knowledge and skills. The results show that most of the teachers perceive themselves as unqualified in terms of measurement and evaluation applications. Kahyaoğlu \& Yangın (2007) determined the views of prospective teachers about professional self-efficacy. This study indicates that PMTs self-efficacy level is lower than other PTs. Azar (2010), compared the levels of pre-service secondary science and mathematics teachers' self-efficacy and analyzed the change of this efficacy according to their demographic characteristics such as gender, graduate university, and major. The results indicate that mathematics teachers' self-efficacy is higher than Physics and Chemistry teachers. Arslan \& Özpınar (2008) determined whether the qualifications required from teachers by primary school programs is in keeping with general teaching competencies provided to pre-service teachers by education faculties. According to results of this study, qualities and competencies required from teachers and PTs are harmonious.

\section{Objectives}

This study aims to explore;

1. The level of self-efficacy of prospective mathematics teachers about competence for planning and organizing instruction,

2. Whether there is a significant difference from the views of faculty, academical success, learning type, interests and knowing the competencies for mathematics teachers or not.

\section{Method}

The descriptive survey method was used for this study. The population of this study is constituted from all PMTs studying at Faculty of Education and Faculty of Science in İnönü University. Total 111 PMTs took place in this research.

"The Scale for Self-efficacy on Competence on Planning and Organizing Instruction" was prepared and used by researcher for this survey. The scale is a kind of check list and constituted from 121 items.

The scale used for this survey;

1. contains all of the sub-competencies related to planning and organizing instruction defined by TNME, in 2009.

2. is harmonious with scales used other surveys trying to explore self efficiacy levels.

The first feature means that the scale has content validity and the second feature means that the scale has adjustment validity. After that, it was examined by experts to get construct validity and done some changes.

The Spearman Brown reliability analysis was used and the internat consistency of the scale was determined 0,998 . So that, the scale is valid and reliable to use for this survey.

\section{Findings}

The findings of the study are presented in the order of the research questions. First of all the characteristics of the sample of this survey is mentioned. Academic success of sample has a normal distribution and most of them are both from Faculty of Science (FS) $(\% 59,5)$ and dual $(\% 72,1)$ students. Also going to cinema $(\% 60,4)$ is the most interested activity for prospective mathematics teachers.

The most effective factors to choose being a mathematics teacher are loving mathematics $(\% 59,5)$, prestige ( $\% 24,3)$, salary $(\% 18,9)$ and orientation of family. On the other side, orientation of friends $(\% 1,8)$ and teachers $(\% 6,3)$ or loving children $(\% 7,2)$ haven't big effect for the sample. The ways for monitoring news by sample are mostly internet $(\% 81,1)$, newspaper $(\% 55,9)$ and TV $(\% 53,2)$ (see appendix (table 1)).

\section{Findings on Use Needs Analysis Techniques}

The analysis revealed that tests $(\% 52,3)$ and natural observation ( $\% 52,3)$ are the leading techniques among the sample. The other dominant techniques are interviews $(44,1)$, literature review $(44,1)$ and job analysis. On the other hand, progel-dacum $(7,2)$ and Delphi $(\% 6,3)$ are the less common techniques. Table 2 presents the frequencies of the sample about using needs analysis techniques. After that, t-test was applied to introduce whether there was relationship between knowledge on subject specific competencies (SSC) and frequencies of needs analysis techniques, or not. Table 3 presents the results. Table 3 shows that there are significant differences about using some needs analysis techniques according to knowledge on SCC. According to this data PTs that know SSC are better about using Progel-Dacum, Delphi, Natural Observations, Interviews and Literature Review techniques than others. So we can say that, PTs that know SSC have a bigger self-efficacy than the others about determining the needs of education. Also, two t-tests were applied to introduce whether there were relationship between knowledge on subject specific competencies (SSC) and firstly faculties and secondly learning types, or not. The results showed there weren't any difference. Also ANOVA was applied to introduce whether there were relationship between Knowledge on SSC and academic success, or not. According to the results, there wasn't any difference, neither. 
Table 2. Frequencies on Use Needs Analysis Techniques

\begin{tabular}{lcccc}
\hline & \multicolumn{2}{c}{ Can Use } & \multicolumn{2}{c}{ Can't Use } \\
\cline { 2 - 5 } Needs Analysis Techniques & $\mathbf{n}$ & $\mathbf{\%}$ & $\mathbf{n}$ & $\mathbf{\%}$ \\
\hline Tests & 58 & 52,3 & 53 & 47,7 \\
Natural Observation T. & 58 & 52,3 & 53 & 47,7 \\
Interview T. & 49 & 44,1 & 62 & 55,9 \\
Literature Review & 49 & 44,1 & 62 & 55,9 \\
Job Analysis T. & 34 & 30,6 & 77 & 69,4 \\
Attitude Scale & 26 & 23,4 & 85 & 76,6 \\
Delphi T. & 7 & 6,3 & 104 & 93,7 \\
Progel-Dacum T. & 8 & 7,2 & 103 & 92,8 \\
\hline
\end{tabular}

Table 3. t-test for Using Needs Analysis Techniques by Knowledge On SSC

\begin{tabular}{cccccccc}
\hline & Knowledge on SSC & f & Mean & Std. Dv. & df & t & p \\
\hline \multirow{2}{*}{ Progel-Dacum } & Yes & 72 & 0,11 &, 316 & 1 & 2,1 & \\
\multirow{2}{*}{ Delphi } & No & 39 & 0,00 &, 000 & & &, $031^{*}$ \\
& Yes & 72 & 0,10 &, 298 & 1 & 2,0 & \\
Tests & No & 39 & 0,00 &, 409 & & &, $045^{*}$ \\
& Yes & 72 & 0,61 &, 491 & 1 & 2,6 & \\
Natural Observation & No & 39 & 0,36 &, 486 & & &, $011^{*}$ \\
& Yes & 72 & 0,61 &, 491 & 1 & 2,5 & \\
Interviews & No & 39 & 0,36 &, 486 & & &, $011^{*}$ \\
& Yes & 72 & 0,54 &, 502 & 1 & 2,9 & \\
Literature Review & No & 39 & 0,26 &, 442 & & &, $003^{*}$ \\
& Yes & 72 & 0,51 &, 503 & 1 & 2,1 & \\
\hline
\end{tabular}

$* \mathrm{p}<0,05$ 
Table 4. Frequencies on Prepare and Use Scale Types

\begin{tabular}{lllll}
\hline \multirow{2}{*}{ Scale Types } & \multicolumn{2}{c}{ Can Use } & \multicolumn{2}{c}{ Can't Use } \\
\cline { 2 - 5 } & $\mathbf{n}$ & $\mathbf{\%}$ & $\mathbf{n}$ & $\mathbf{\%}$ \\
\hline Observation forms & 43 & 38,7 & 68 & 61,3 \\
Leisure time activities & 43 & 38,7 & 68 & 61,3 \\
Attention tests & 41 & 36,9 & 70 & 63,1 \\
Scale of reason for failure & 39 & 35,1 & 72 & 64,9 \\
Social adjustment tests & 34 & 30,6 & 77 & 69,4 \\
Test anxiety s. & 34 & 30,6 & 77 & 69,4 \\
Study habits assessment s. & 32 & 28,8 & 79 & 71,2 \\
Self-assessment questionnaire & 31 & 27,9 & 80 & 72,1 \\
Peer appraisal & 30 & 27,0 & 81 & 73,0 \\
Interest tests & 30 & 27,0 & 81 & 73,0 \\
Critics questionnaire & 28 & 25,2 & 83 & 74,8 \\
Anxiety level s. & 27 & 24,3 & 84 & 75,7 \\
Parents assessment s. & 26 & 23,4 & 85 & 76,6 \\
Happiness s. & 25 & 22,5 & 86 & 77,5 \\
Memory tests & 23 & 20,7 & 88 & 79,3 \\
Guess who questionnaire & 18 & 20,7 & 88 & 79,3 \\
Sosyometry s. & 23 & 18,9 & 90 & 81,1 \\
Perceived family support s. & 21 & 17,1 & 92 & 82,9 \\
Academic self-concept s. & 19 & 17,1 & 92 & 82,9 \\
Self-directed learning readiness s. & 19 & 16,2 & 93 & 83,8 \\
Vocational maturity s. & & 14,4 & 95 & 85,6 \\
\hline
\end{tabular}

\section{Findings on Indicators about Prepare and Use Scales}

The analysis revealed that observation forms $(\% 38,7)$ and leisure time activities questionnaire $(\% 38,7)$ are the leading form types among the sample. On the other hand, vocational maturity scales $(\% 14,4)$, self-directed learning readiness scales $(\% 16,2)$ and perceived family support scales $(17,1)$ are the less common techniques. Table 4 presents the frequencies of the sample about preparing and using scales. After that, t-tests were applied to introduce whether there were relationship between preparing and using scale types between firstly faculties, secondly learning type and thirdly knowledge on SCC, or not. According to these t-tests there wasn't any difference between groups. Also ANOVA was applied to introduce whether there were relationship between preparing and using scale types and academic success, or not. According to the results, there wasn't any difference, neither.

\section{Findings on Use Learning Materials}

The analysis revealed that books $(\% 78,4)$, graphics $(\% 64)$, geometrical shapes $(\% 61,3)$ and blackboard are the leading materials among the sample. The other dominant materials are $3 \mathrm{D}$ models $(\% 59,5)$ and geometry boards $(\% 49,5)$. On the other hand, cubic set $(\% 13,5)$, decoration set $(\% 14,4)$, transparent fraction cards $(\% 14,4)$ and square set $(\% 15,3)$ are the less common techniques. Table 5 presents the frequencies of the sample about using learning materials. T-test was applied to introduce whether there was relationship between using learning materials and faculties, or not. Table 6 presents the results. According to table 6 , there are differences between FE and FS nearly for all materials. The differences are in FE's favor. This results shows that PTs from FE have bigger self-efficacy than from FS. T-test also was applied to introduce whether there was relationship between using learning materials and knowledge on SSC, or not. Table 5 presents the results. Table 5 shows that there are significant differences about using some learning materials according to knowledge on SCC. According to this data PTs that know SSC are better about using isometric paper, 3D-models, punctuated paper, models and samples, geometry boards, fraction bars, tangram, blackboards than others. So we can say that, PTs that know SSC have a bigger self-efficacy than the others about using learning materials. After that, t-tests was applied to introduce whether there were relationship between preparing and using scale types between learning types, or not. According to the t-test there wasn't any difference between groups. 
Table 5. Frequencies on Use Learning Materials

\begin{tabular}{|c|c|c|c|c|}
\hline \multirow{2}{*}{ Learning Materials } & \multicolumn{2}{|c|}{ Can Use } & \multicolumn{2}{|c|}{ Can't Use } \\
\hline & $\mathrm{f}$ & $\%$ & $\mathrm{f}$ & $\%$ \\
\hline Volume set & 41 & 36,9 & 70 & 63,1 \\
\hline Unit Cubes & 36 & 32,4 & 75 & 67,6 \\
\hline Isometric paper & 30 & 27,0 & 81 & 73,0 \\
\hline Hexagonal paper & 20 & 18,0 & 91 & 82,0 \\
\hline 3-D models & 66 & 59,5 & 45 & 40,5 \\
\hline Pattern blocks & 23 & 20,7 & 88 & 79,3 \\
\hline Punctuated paper & 39 & 35,1 & 72 & 64,9 \\
\hline Square set & 17 & 15,3 & 94 & 84,7 \\
\hline Models and samples & 40 & 36,0 & 71 & 64,0 \\
\hline Symmetry mirror & 37 & 33,3 & 74 & 66,7 \\
\hline Octagonal paper & 19 & 17,1 & 92 & 82,9 \\
\hline Algebric diamonds & 19 & 17,1 & 92 & 82,9 \\
\hline Maps & 46 & 41,4 & 65 & 58,6 \\
\hline Geometry Boards & 55 & 49,5 & 56 & 50,5 \\
\hline Rectanglar Paper & 31 & 27,9 & 80 & 72,1 \\
\hline Cube set & 15 & 13,5 & 96 & 86,5 \\
\hline Diagram & 43 & 38,7 & 68 & 61,3 \\
\hline Fractional bars & 37 & 33,3 & 74 & 66,7 \\
\hline Triangular paper & 33 & 29,7 & 78 & 70,3 \\
\hline Decoration set & 16 & 14,4 & 95 & 85,6 \\
\hline Graphics & 71 & 64,0 & 40 & 36,0 \\
\hline Tangram & 21 & 18,9 & 90 & 81,1 \\
\hline Rhombus paper & 34 & 30,6 & 77 & 69,4 \\
\hline Blackboard & 68 & 61,3 & 43 & 38,7 \\
\hline Cartoons & 29 & 26,1 & 82 & 73,9 \\
\hline Trensparent fraction cards & 16 & 14,4 & 95 & 85,6 \\
\hline Punctuated and circular paper & 22 & 19,8 & 89 & 80,2 \\
\hline Base 10 blokcs & 32 & 28,8 & 79 & 71,2 \\
\hline Books & 87 & 78,4 & 24 & 21,6 \\
\hline Geometric shapes & 68 & 61,3 & 43 & 38,7 \\
\hline Circular paper & 24 & 21,6 & 87 & 78,4 \\
\hline
\end{tabular}


Table 6. T-test for Use Learning Materials by Faculties

\begin{tabular}{|c|c|c|c|c|c|c|c|}
\hline & Faculty & f & Mean & Std. Dv. & df & $\mathrm{t}$ & $\mathbf{p}$ \\
\hline \multirow{2}{*}{ Volume set } & FE & 45 & 0,51 & ,506 & \multirow{2}{*}{109} & \multirow{2}{*}{2,551} & \multirow{2}{*}{,012 } \\
\hline & FS & 66 & 0,27 & ,449 & & & \\
\hline \multirow{2}{*}{ Unit cubes } & $\mathrm{FE}$ & 45 & 0,49 & ,506 & \multirow{2}{*}{109} & \multirow{2}{*}{3,047} & \multirow{2}{*}{,003 } \\
\hline & FS & 66 & 0,21 & ,412 & & & \\
\hline \multirow{2}{*}{ Isometric paper } & $\mathrm{FE}$ & 45 & 0,44 & ,503 & \multirow{2}{*}{109} & \multirow{2}{*}{3,362} & \multirow{2}{*}{, 001} \\
\hline & FS & 66 & 0,15 & ,361 & & & \\
\hline \multirow{2}{*}{ Hexagonal paper } & $\mathrm{FE}$ & 45 & 0,29 & ,458 & \multirow{2}{*}{109} & \multirow{2}{*}{2,336} & \multirow{2}{*}{, 022} \\
\hline & FS & 66 & 0,11 & ,310 & & & \\
\hline \multirow{2}{*}{ 3D models } & $\mathrm{FE}$ & 45 & 0,76 & ,435 & \multirow{2}{*}{109} & \multirow{2}{*}{3,019} & \multirow{2}{*}{, 003} \\
\hline & FS & 66 & 0,48 & ,504 & & & \\
\hline Pattern blocks & $\mathrm{FE}$ & 45 & 0,38 & , 490 & 100 & 3527 & 001 \\
\hline 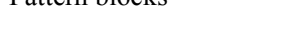 & FS & 66 & 0,09 & ,290 & 109 & ( & \\
\hline Punctuated paper & $\mathrm{FE}$ & 45 & 0,60 & ,495 & & & \\
\hline Punctuated paper & FS & 66 & 0,18 & 389 & 109 & 4,752 &, 000 \\
\hline Square set & $\mathrm{FE}$ & 45 & 0,27 & ,447 & 100 & 560 & 012 \\
\hline Square set & FS & 66 & 0,08 & ,361 & 109 & 2,569 & ,012 \\
\hline Models and camnles & $\mathrm{FE}$ & 45 & 0,67 & ,477 & 100 & 6145 & 000 \\
\hline Noders and sampies & FS & 66 & 0,15 & ,361 & 109 & 0,145 &, 000 \\
\hline Symmetry mirror & $\mathrm{FE}$ & 45 & 0,49 & ,506 & 100 & 2858 & 005 \\
\hline symmerry mirror & FS & 66 & 0,23 & ,422 & 109 & 2,858 & , \\
\hline Octaonal naner & $\mathrm{FE}$ & 45 & 0,29 &, 458 & 100 & 2569 & 012 \\
\hline & FS & 66 & 0,09 & ,290 & & & \\
\hline & $\mathrm{FE}$ & 45 & 0,67 & ,477 & 109 & 4.780 & 000 \\
\hline Maps & FS & 66 & 0,24 & ,432 & 109 & $4, / 80$ & ,000 \\
\hline Geometry hoards & $\mathrm{FE}$ & 45 & 0,67 & 477, & 100 & 3077 & 003 \\
\hline Geometry boards & FS & 66 & 0,38 & ,489 & 109 & $3,0 / 1$ & , \\
\hline Pectanolar noner & $\mathrm{FE}$ & 45 & 0,42 & ,499 & 100 & 2716 & 008 \\
\hline Kectangrar paper & FS & 66 & 0,18 &, 389 & 109 & $2, / 10$ &, 000 \\
\hline Cuhe sets & $\mathrm{FE}$ & 45 & 0,24 &, 435 & 100 & 2581 & 012 \\
\hline Cuoe sets & FS & 66 & 0,06 & ,240 & 109 & 2,501 & \\
\hline Diagram & $\mathrm{FE}$ & 45 & 0,58 & ,499 & 109 & 3,476 & 001 \\
\hline & FS & 66 & 0,26 & ,441 & 109 & & \\
\hline Fraction hars & $\mathrm{FE}$ & 45 & 0,47 & ,505 & 100 & 2435 & 017 \\
\hline Fraction bars & FS & 66 & 0,24 & ,432 & 109 & 2,435 & (017 \\
\hline Trianoular naner & $\mathrm{FE}$ & 45 & 0,44 & ,503 & 100 & 750 & 007 \\
\hline Irlangular paper & FS & 66 & 0,20 & ,401 & 109 & 2,159 & , \\
\hline Graphics & $\mathrm{FE}$ & 45 & 0,78 & ,420 & 100 & 2640 & 010 \\
\hline Urapnies & FS & 66 & 0,55 &, 502 & 109 & 2,040 &, 010 \\
\hline Tanoram & $\mathrm{FE}$ & 45 & 0,31 & ,468 & 100 & 2577 & 012 \\
\hline I angram & FS & 66 & 0,11 & ,310 & 109 & 2,011 &, 012 \\
\hline Cartoons & $\mathrm{FE}$ & 45 & 0,42 & ,499 & 100 & 3121 & 003 \\
\hline & FS & 66 & 0,15 & ,361 & & & \\
\hline Trancnarent fraction cards & $\mathrm{FE}$ & 45 & 0,27 & ,447 & 100 & 2825 & 006 \\
\hline Transparent fraction cards & FS & 66 & 0,06 & 240 & 109 & 2,825 & (2006 \\
\hline Punctuated circular noner & $\mathrm{FE}$ & 45 & 0,36 & ,484 & 100 & 3288 & 002 \\
\hline Punctuated circular paper & FS & 66 & 0,09 & ,290 & 109 & 3,288 & , \\
\hline Rase 10 blocks & $\mathrm{FE}$ & 45 & 0,42 & ,499 & 100 & $2522+20$ & 014 \\
\hline Dase 10 olocks & FS & 66 & 0,20 & ,401 & 109 & 2,522 &, 014 \\
\hline Circular naner & $\mathrm{FE}$ & 45 & 0,33 & ,477 & 100 & 2378 & 020 \\
\hline Crrcurar paper & FS & 66 & 0,14 & ,346 & 109 & $2,3 / 0$ &, $0<0$ \\
\hline
\end{tabular}

$* \mathrm{p}<0,05$ 
Table 7. T-test on Use Learning Materials by Knowledge on SSC

\begin{tabular}{|c|c|c|c|c|c|c|c|}
\hline Learning materials & $\begin{array}{c}\text { Knowledge on } \\
\text { SSC }\end{array}$ & f & Mean & Std. Dv. & df & $\mathbf{t}$ & $\mathbf{p}$ \\
\hline \multirow{2}{*}{ Isometric paper } & Yes & 72 & 0,35 & 0,479 & \multirow{2}{*}{109} & \multirow{2}{*}{2,529} & \multirow{2}{*}{, 013} \\
\hline & No & 39 & 0,13 & 0,339 & & & \\
\hline \multirow{2}{*}{ 3D models } & Yes & 72 & 0,68 & 0,470 & \multirow{2}{*}{109} & \multirow{2}{*}{2,557} & \multirow{2}{*}{, 012} \\
\hline & No & 39 & 0,44 & 0,502 & & & \\
\hline \multirow{2}{*}{ Punctuated paper } & Yes & 72 & 0,43 & 0,428 & \multirow{2}{*}{109} & \multirow{2}{*}{2,416} & \multirow{2}{*}{, 017} \\
\hline & No & 39 & 0,21 & 0,366 & & & \\
\hline \multirow{2}{*}{ Models and samples } & Yes & 72 & 0,43 & 0,499 & \multirow{2}{*}{109} & \multirow{2}{*}{2,116} & \multirow{2}{*}{, 037} \\
\hline & No & 39 & 0,23 & 0,427 & & & \\
\hline \multirow{2}{*}{ Geomtry board } & Yes & 72 & 0,60 & 0,494 & \multirow{2}{*}{109} & \multirow{2}{*}{3,003} & \multirow{2}{*}{, 003} \\
\hline & No & 39 & 0,31 & 0,468 & & & \\
\hline \multirow{2}{*}{ Fraction Bars } & Yes & 72 & 0,42 & 0,496 & \multirow{2}{*}{109} & \multirow{2}{*}{2,583} & \multirow{2}{*}{, 011} \\
\hline & No & 39 & 0,18 & 0,389 & & & \\
\hline \multirow{2}{*}{ Tangram } & Yes & 72 & 0,25 & 0,436 & \multirow{2}{*}{109} & \multirow{2}{*}{2,253} & \multirow{2}{*}{, 026} \\
\hline & No & 39 & 0,08 & 0,270 & & & \\
\hline \multirow{2}{*}{ Blackboards } & Yes & 72 & 0,71 & 0,458 & \multirow{2}{*}{109} & \multirow{2}{*}{2,892} & \multirow{2}{*}{, 005} \\
\hline & No & 39 & 0,44 & 0,502 & & & \\
\hline
\end{tabular}

$* \mathrm{p}<0,05$

\section{Findings on Variables that are Thought to be Used at Courses}

The variables that are thought to be used at courses by prospective mathematics teachers were investigated. The analysis revealed that question banks ( $\% 89,2)$, question paper $(\% 83,8)$, teacher's handbook $(\% 68,5)$ and intelligence games $(\% 68,5)$ are the leading variables among the sample. On the other hand, songs $(\% 7,2)$, newspapers $(\% 16,2)$ and magazines $(\% 26,1)$ are the less common variables. Table 8 presents the frequencies of the sample about variables that are thought to be used at courses. T-test was applied to introduce whether there was relationship between variables thought to be used at courses and faculties, or not. Table 9 presents that there is difference between PMTs that know and don't know SSC according to some variables. After that, $\mathrm{t}$-tests were applied to introduce whether there were relationship between variables thought to be used and firstly faculties and secondly learning type, or not. According to these t-tests there wasn't any difference between groups.

Table 8. Frequencies about Variables are thought to be Used at Courses

\begin{tabular}{lcccc}
\hline \multirow{2}{*}{ Variables thought to be used at courses } & \multicolumn{2}{c}{ Can Use } & \multicolumn{2}{c}{ Can't Use } \\
\cline { 2 - 5 } & $\mathbf{N}$ & $\mathbf{\%}$ & $\mathbf{n}$ & $\mathbf{\%}$ \\
\hline Question Banks & 99 & 89,2 & 12 & 10,8 \\
Question Papers & 93 & 83,8 & 18 & 16,2 \\
Teacher's Handbook & 76 & 68,5 & 35 & 31,5 \\
Intelligence Games & 76 & 68,5 & 35 & 31,5 \\
Operation Games & 63 & 56,8 & 48 & 43,2 \\
Educational CDs & 53 & 47,7 & 58 & 52,3 \\
Web Sites & 51 & 45,9 & 66 & 54,1 \\
Crosswords & 44 & 39,6 & 67 & 60,4 \\
Magazines & 29 & 26,1 & 82 & 73,9 \\
Newspaper & 18 & 16,2 & 93 & 83,8 \\
Songs & 8 & 7,2 & 103 & 92,8 \\
\hline
\end{tabular}


Table 9. $\mathrm{t}$-test for Variables Thought to be Used at Courses by Knowledge on SSC

\begin{tabular}{|c|c|c|c|c|c|c|c|}
\hline & K. on SSC & f & Mean & Std. Dv. & df & $\mathbf{t}$ & $\mathbf{p}$ \\
\hline \multirow{2}{*}{ Teacher's handbook } & Yes & 72 & 0,75 & 0,436 & \multirow{2}{*}{109} & \multirow{2}{*}{2,031} & \multirow{2}{*}{, 045} \\
\hline & No & 39 & 0,56 & 0,502 & & & \\
\hline \multirow{2}{*}{ Magazines } & Yes & 72 & 0,33 & 0,475 & \multirow{2}{*}{109} & \multirow{2}{*}{2,387} & \multirow{2}{*}{, 019} \\
\hline & No & 39 & 0,13 & 0,339 & & & \\
\hline \multirow{2}{*}{ Question papers } & Yes & 72 & 0,89 & 0,316 & \multirow{2}{*}{109} & \multirow{2}{*}{2,000} & \multirow{2}{*}{, 048} \\
\hline & No & 39 & 0,74 & 0,442 & & & \\
\hline \multirow{2}{*}{ Web sites } & Yes & 72 & 0,54 & 0,502 & \multirow{2}{*}{109} & \multirow{2}{*}{2,401} & \multirow{2}{*}{, 018} \\
\hline & No & 39 & 0,31 & 0,468 & & & \\
\hline \multirow{2}{*}{ Newspaper } & Yes & 72 & 0,22 & 0,419 & \multirow{2}{*}{109} & \multirow{2}{*}{2,370} & \multirow{2}{*}{, 020} \\
\hline & No & 39 & 0,05 & 0,223 & & & \\
\hline
\end{tabular}

$* \mathrm{p}<0,05$

Table 10. Frequencies on Use Teaching Methods

\begin{tabular}{lcccc}
\hline \multirow{2}{*}{ Teaching Methods } & \multicolumn{2}{c}{ Can Use } & \multicolumn{2}{c}{ Can't Use } \\
\cline { 2 - 5 } & $\mathrm{f}$ & $\%$ & $\mathrm{f}$ & $\%$ \\
\hline Lecture & 96 & 86,5 & 15 & 13,5 \\
Problem Solving & 92 & 82,9 & 19 & 17,1 \\
Demonstration & 78 & 70,3 & 33 & 29,7 \\
Questioning & 77 & 69,4 & 34 & 30,6 \\
Discussion & 58 & 52,3 & 53 & 47,7 \\
Computer-Based Teaching & 57 & 51,4 & 54 & 48,6 \\
Brain storming & 53 & 47,7 & 58 & 52,3 \\
Cooperative learning & 43 & 38,7 & 68 & 61,3 \\
Case study & 41 & 36,9 & 70 & 63,1 \\
Team teaching & 35 & 31,5 & 76 & 68,5 \\
Project based teaching & 31 & 27,9 & 80 & 72,1 \\
Programmed learning & 31 & 27,9 & 80 & 72,1 \\
Educational games & 30 & 27,0 & 81 & 73,0 \\
Story telling method & 29 & 26,1 & 82 & 73,9 \\
Role playing & 22 & 19,8 & 89 & 80,2 \\
Creative drama & 21 & 18,9 & 90 & 81,1 \\
Aquarium & 15 & 13,5 & 96 & 86,5 \\
Description & 13 & 11,7 & 98 & 88,3 \\
\hline
\end{tabular}

\section{Findings on Use Teaching Methods}

Using teaching methods by prospective mathematics teachers were investigated. The analysis revealed that lecture method $(\% 86,5)$, problem solving $(\% 82,9)$, demonstration $(\% 70,35)$ and questioning $(\% \% 69,4)$ are the leading variables among the sample. On the other hand, description method $(\% 11,7)$, aquarium $(\% 13,5)$ and role playing methods $(\% 19,8)$ are the less common variables. Table 10 presents the frequencies of the sample about using teaching methods. T-test was applied to introduce whether there was relationship between teaching methods and faculties, or not. Table 11 presents the results. According to table 11, there are differences between FE and FS for questioning, storytelling and team teaching methods. The differences are in FE's favor. This results shows that PTs from FE have bigger self-efficacy than from FS about using some teaching methods. Then, t-test also was applied to introduce whether there was relationship between using learning materials and knowledge on SSC, or not. Table 12 presents the results. Table 12 shows that there are significant differences about using some teaching methods according to learning types. According to this data PTs that from D.L. are better about using aquarium, description and storytelling methods than from N.L. So we can say that, PTs that are from D.L. have a bigger self-efficacy than from N.L. about using learning materials. T-test also was applied to introduce whether there was relationship between teaching methods and knowledge on SCC, or not. Table 13 presents the results. According to table 13, there are differences between the PTs that know SCC and don't know SCC for Computer based learning and questioning methods. The differences are in the PTs know SCC's favor. This result shows that PTs know SCC have bigger self-efficacy than don't know SCC. 
Table 11. T-test for Using the Teaching Methods According to Faculties

\begin{tabular}{lccccccc}
\hline & Faculties & $\mathbf{f}$ & $\mathbf{\%}$ & Std. Dev. & df & $\mathbf{t}$ & $\mathbf{p}$ \\
\hline \multirow{2}{*}{ Question and answer } & FE & 45 &, 51 &, 506 & & & \\
& FS & 66 &, 27 &, 449 & 109 & 2,551 &, 012 \\
Story telling & FE & 45 &, 49 &, 506 & & & \\
\multirow{2}{*}{ Team teaching } & FS & 66 &, 21 &, 412 & 109 & 3,047 &, 003 \\
& FE & 45 &, 44 &, 503 & & & \\
\hline
\end{tabular}

${ }^{*} \mathrm{p}<0,05$

Table 12. T-Test for Using the Teaching Methods According to Learning Types

\begin{tabular}{lccccccc}
\hline & Learning Type & $\mathbf{f}$ & $\mathbf{\%}$ & Std. Dev. & $\mathbf{d f}$ & $\mathbf{t}$ & $\mathbf{p}$ \\
\hline \multirow{2}{*}{ Aquarium } & N.L. & 31 &, 00 &, 000 & & & \\
& D.L. & 80 &, 19 &, 393 & 109 & $-2,650$ &, 009 \\
\multirow{2}{*}{ Description } & N.L. & 31 &, 00 &, 000 & & & \\
& D.L. & 80 &, 16 &, 371 & 109 & $-2,430$ &, 017 \\
\multirow{2}{*}{ Storytelling } & N.L. & 31 &, 10 &, 301 & & & \\
& D.L. & 80 &, 33 &, 371 & & $-2,502$ &, 014 \\
\hline
\end{tabular}

$* \mathrm{p}<0,05$

Table 13. T-test for Using Methods According To Knowledge On SCC

\begin{tabular}{|c|c|c|c|c|c|c|c|}
\hline & K. on SCC & f & $\%$ & Std. Dev. & df & $\mathbf{t}$ & $\mathbf{p}$ \\
\hline \multirow{2}{*}{ Computer based learning } & Yes & 72 &, 58 & ,496 & \multirow{2}{*}{109} & \multirow{2}{*}{2,023} & \multirow{2}{*}{, 047} \\
\hline & No & 39 & ,38 & ,493 & & & \\
\hline \multirow{2}{*}{ Question and answer } & Yes & 72 &, 81 & ,399 & \multirow{2}{*}{109} & \multirow{2}{*}{3,646} & \multirow{2}{*}{, 000} \\
\hline & No & 39 & ,49, &, 506 & & & \\
\hline
\end{tabular}

$\mathrm{p}<0,05$

\section{Conclusions}

The sample's interests are mostly going to cinema, listening to music, internet, doing sports. This result indicates that PTs perceived university as an entertainment center and didn't interest in educational activities such as vocational, mathematical and general culture knowledge.

The most important factor to choose teaching mathemtics is loving mathematics. This is normal but on the other hand the level of loving children isn't high enough. This result indicates that PTs thinks that doing mathematics is same teaching mathematics. Loving mathematics requires to be a mathematician more than a mathematics teacher. This result could be arose from the job opportunities.

$\% 35,1$ percent of the sample doesn't know what SSC were. This is a big rate for PTs. This result indicates that PTs didn't monitore news about teaching and learning area. This also shows that there wasn't enough cooperation between TNME and universities about to share activities.

Teachers don't know enough needs analysis techniques.
This result indicates that teachers aren't good enough at curriculum development. On the other hand, teachers believe that they are good at interview and natural observation techniques. The techniques don't have technical terms and PTs think that they were able to do what they could understand its name. The low rate of progel-dacum and delphi supports this result.

Some of the PTs $(\% 16,2)$ aren't able to prepare and use any scales. The PTs that are able to use more than 5 scales are only 36 percent. Modern teaching methods needs to take into consideration the individual differences. So, teachers are neccessary to use diagnostic scales. And the low level of using them by PTs indicates that they weren't able to define students enough as an educator.

The results indicate that PTs are able to use the teacher centered learning materials. This preference can arise from not ot $b$ well educated about the constructivist learning materials such as cubes set, squares set, trensparent fraction bars.

According to the results, PTs from FE have a bigger self 
efficacy than FS about using learning materials. This result can arise from the difference between the teaching ability of teaching stuffs.

The data obtained from the survey indicates that PTs thought to use mostly question banks and question papers. This result arise from the central examinations. The believe of passing central examinations thanks to special courses that are based to solving questions makes PTs to incite using question banks and question papers.

The most frequently chosen teaching method by PT was lecture method. This result indicates that PTs didn't think that they were not able to use constructivist approach's teaching method, yet. This can arise from the hardness of other methods. Also, being mathematicians more than mathematics teachers could make the sample to like keeping away from interactions with students. Thinking to be equal of being a mathematician and a mathematics teacher can cause this result. According to the table 10, PTs think that they were good at problem solving method. Mathematics courses are appropriate to solving problems and questions. But, this result may cause from misconception. Problem solving method needs applications and being original but on the other hand solving question can be applied with pencil and paper tests. This differences must be misconcepted by PTs may created this result.

According to faculties, PTs have a difference from each other. This differences seen on the methods needs more interaction such as question and answer method, storytelling method and team teaching.

There was difference between NL and DL PTs. This may cause from the teaching stuff differences of them. Senior teaching stuff mostly choose DL courses and this may cause being better DL than NL, according to some methods.

\section{Suggestions}

Perceiving universities as an entertainment center by students makes them to fail their educational goals. Passing central examinations is so hard that the students feel sluggish when they started to university. Then they look for new interests. During this lassitude period, teaching stuff may apply them vocational guidence on vocational information, SSC of mathematics and general culture. Teaching stuff explain to students what the news are and the subjects that need to be studied.

Not to love children is a big problematic for prospective students. Due to the importance of affective entry characteristics for courses, not to love children will make PTs one apice of mathematician. This isn't required for teaching training institues. Making the PTs to read books that describe the innerworld of children may help them to like children.

It was seen from the survey, 35.1 percent of the sample didn't know what SSC was. This is a high rate for PTs. This result indicates that universities aren't good at to succeed their goals as much as PTs. Monitoring the web sites of
TNME, CHE, Measurement-Selection and Placement Center (MSPC) and The Scientific and Technological Research Council of Turkey (STRCT) that direct the educational events may help students to update their knowledge about education. Also, mentioning news at courses may also solve this problem.

Needs analysis is the most important step for developing curriculum. In this respect, needs analysis must be emphasized and explored the details by teaching stuff on Curriculum Development courses.

Also, the goals of all courses may be declared by teaching stuff to make students aware of the importance of the goals.

The result about using scales indicates that PTs don't attach importance to individual differences. This result may cause from the sample's own experiences. In this respect, preparing educational environments of PTs according to their individual differences may help them to aware of the importance of individual differences. Giving different homework types to different PTs and encourage them to participate different extra-curricular activities due to their abilities make help to solve this problematic.

Also, supplying feed backs to students about the questionnaires and scales and arranging courses according to results, may make PTs feel the usefulness of them.

Being not enough to use learning materials of PTs is the fault of teacher training institutes. All of the learning materials that can be used at mathematics courses must be introduced by teaching stuff to PTs. Also, using learning materials in courses at universities may also help PTs to perceive the importance of using materials.

The results about using the teaching method indicate that, PTs don't like to get interaction with students. But, as it emphasized before, being mathematics teacher needs to get interaction with students. PTs must review themselves and make a decision about their job. On the other hand, teaching stuff can cooperate PTs at courses. Group projects, team teaching, discussion, educational games, role playing may help the PTs to get socialized and may use them at their courses too.

Researchers that intend to study teacher-efficacy can investigate the teachers' efficacy level, what teachers need to be more efficient, what can be done to prepare PTs for more effective.

\section{Discussions}

The survey, that investigated the level of self-efficacy about the competence of planning and organizing instruction of PMTs in İnönü University, indicated that PTs weren't well enough about some performance indicators. The PTs have not to be able to reach to a lot of performance indicators, yet. Wrong preference about vocations and teaching activities at universities may cause this result. Vocational guidance must be held in Turkey and PTs must perceive the importance of interactions for teaching. Also universities may cooperate with TNME and STRCT to make PTs to be aware of news about education. 


\section{APPENDIX}

Tables

Table 1. Characteristics of Sample

\begin{tabular}{|c|c|c|c|c|c|}
\hline & $\mathbf{N}$ & $\%$ & & $\mathbf{N}$ & $\%$ \\
\hline \multicolumn{3}{|l|}{ Academic Success } & \multicolumn{3}{|l|}{ Interests } \\
\hline$\ldots \ldots-59,49$ & 4 & 3,6 & Cinema & 67 & 60,4 \\
\hline $60-64,49$ & 27 & 24,3 & Music & 61 & 55 \\
\hline $65-69,49$ & 34 & 28,7 & Internet & 55 & 49,5 \\
\hline $70-74,49$ & 25 & 22,5 & Sports & 51 & 45,9 \\
\hline $75-79,49$ & 14 & 11,6 & Book & 47 & 42,3 \\
\hline $80-\ldots$ & 7 & 6,3 & Science and Technology & 45 & 40,5 \\
\hline \multicolumn{3}{|c|}{ Factors to Choose Being a Mathematics Teacher. } & \multicolumn{3}{|c|}{ Knowledge on Subject Specific Competencies of Mathematics } \\
\hline Loving Mathematics. & 66 & 59,5 & Yes & 72 & 64,9 \\
\hline Prestige & 27 & 24,3 & No & 39 & 35,1 \\
\hline Salary & 21 & 18,9 & \multicolumn{3}{|l|}{ Ways for Monitoring News } \\
\hline Orientation of Family & 18 & 16,2 & Newspaper & 62 & 55,9 \\
\hline Loving old Mathematics Teachers & 9 & 8,1 & Radio & 7 & 6,3 \\
\hline Loving Children & 8 & 7,2 & Periodical & 38 & 34,2 \\
\hline Orientation of Teachers & 7 & 6,3 & $\mathrm{TV}$ & 59 & 53,2 \\
\hline Orientation of Friends & 2 & 1,8 & Internet & 90 & 81,1 \\
\hline \multicolumn{3}{|l|}{ Faculty } & None & 3 & 2,7 \\
\hline Faculty of Education & 45 & 40,5 & \multicolumn{3}{|l|}{ Learning Type } \\
\hline \multirow[t]{2}{*}{ Faculty of Science } & 66 & 59,5 & Normal & 31 & 27,9 \\
\hline & & & Dual & 80 & 72,1 \\
\hline
\end{tabular}

[7] İlhan, A.Ç., (2004). 21. yüzyılda öğretmen yeterlikleri. Bilim ve Aklın Aydınlığında Eğitim Dergisi, yı1 5, s.58.

\section{REFERENCES}

[1] Bandura, A., (1977). Self-Efficacy: Toward a unifying theory of behavioral change. Psychogical Review, 84(2)191-215

[2] Bandura, A. (1994). Self-efficacy, In V.S. Ramachaudran(Ed.), Encyclopedia of Human Behavior. (4), 71-81. Newyork: Academic Press.

[3] DPT, (2000). Yükseköğretim. Özel İhtisas Komisyonu Raporu: Sekizinci Beş Yıllık Kalkınma Planı, Public No. DPT:2534, Ankara.

[4] Enochs L. G., and Riggs, I. M., (1990). Further Development of an Elementary Science Teaching Efficacy Belief Instrument: A Preservice Elementary Scale, School Science and Mathematics, 90(8), 694-706

[5] Gibson, S., ve Dembo, M. H. (1984). Teacher efficacy: A construct validation. Journal of Educational Psychology, 76(4), 569-582.

[6] Gürkan, T., Duman, T., Güneysu, S., Yalın, H.İ., Olkun, S., Bıkmaz, F. vd. (2004). Öğretmen yeterliği taslağında yer alan yeterlik alanları. Bilim ve Aklın Aydınlığında Eğitim Dergisi, y11 5, say1 58.

[8] MEB-ÖYEGM (2009). Ortaöğretim matematik öğretmeni özel alan yeterlikleri. 01.02.2010 tarihinde Öğretmen Yetiştirme ve Eğitimi Genel Müdürlügü: http://otmg.meb.gov.tr/yet.pdf2/matematik07\%5B1\%5D.08. 2009.pdf adresinden alınd1.

[9] Özkan, Ö., Tekkaya, C., Çakıroğlu, J. (2002). Fen Bilgisi Aday Öğretmenlerin Fen Kavramlarını Anlama Düzeyleri. Fen Öğretimine Yönelik Tutum ve Öz-yeterlik İnançları, V. Fen Bilmleri Eğitimi Kongresi, ODTÜ, Ankara.

[10] Özkan,R., (2004). Öğretmen yeterlikleri üzerine baz1 düşünceler. Bilim ve Aklın Aydınlığında Eğitim Dergisi, yıl 5, s.58

[11] Scholz, U., Dona, B.G., Sud, A., Schwarzer, R., (2002). Is general self-efficacy a universal construct?. European Journal of Psychological Assessment, 18 (3), 242-251.

[12] Seferoğlu, S.S., (2004). Öğretmen yeterlikleri ve mesleki gelişim. Bilim ve Aklın Aydınlığında Eğitim Dergisi, yıl 5, s. 58

[13] Şahin, A.E., (2004). Öğretmen yeterliklerinin belirlenmesi. Bilim ve Aklın Aydınlığında Eğitim Dergisi, yıl 5, s.58

[14] Tschannen -Moran, M., Woolfolk-Hoy, A., ve Hoy, W. K., (1998). Teacher efficacy: Its meaning and measure. Review 
of Educational Research, 68, 202-248.

[15] Yavuzer, Y., Koç, M. (2002). Eğitim Fakültesi Öğrencilerinin Öğretmen Yetkinlikleri Üzerinde Bir Değerlendirme. Niğde Üniversitesi Eğitim Fakültesi Dergisi. 1 (1), 35-43

[16] Yılmaz, M., Köseoğlu, P., Gerçek, C. ve Soran,H. (2004). Öğretmen öz-yeterlik inancı. Bilim ve Aklın Aydınlığında Eğitim Dergisi, yıl 5, s.58.

[17] Şeker, H., Deniz, S. \& Görgen, İ. (2005). Prospective teachers' assessment of teacher competencies. Kuram ve Uygulamada Eğitim Yönetimi, 42, 237-253.

[18] Çakan, M. (2004). Comparison of elementary and secondary school teachers in terms of their assessment practices and perceptions toward their qualification levels. Ankara
University, Journal of Faculty of Educational Sciences, 37 (2), 99-114.

[19] Kahyaoğlu, M. \& Yangın, S. (2007). Views of prospective teachers in elementary school teaching departments about professional self-efficacy. Kastamonu Education Journal, 15 (1), 73-84.

[20] Azar, A. (2010). In-service and pre-service secondary science teachers' self-efficacy beliefs about teaching. ZKU Journal of Social Sciences, 6 (12), 235-252.

[21] Arslan, S. \& Özpınar, İ. (2008). Teacher qualifications: Comparison between primary school curriculum expectations and teachers acquisitions in education faculties. Necatibey Faculty of Education Electronic Journal of Science and Mathematics Education, 2 (1), 38-63 